\title{
Operant control of surface body temperature
}

\author{
S. THOMAS ELDER and KATHLEEN G. FRENTZ \\ University of New Orleans, New Orleans, Louisiana 70122
}

\begin{abstract}
- Eighteen male volunteer college students, 18 to 28 years old, participated in an experiment to determine if humans could be trained to change the surface temperature of their right hand and, if so, to determine the specificity of the response. Training occurred over two successive 1-h daily sessions, and feedback consisted of a digital display. The results showed that subjects could learn to increase/decrease surface temperature under conditions of contingent feedback/ reinforcement. Further, the response was somewhat specific and no general autonomic changes were observed to accompany the acquired temperature change.
\end{abstract}

With few exceptions (e.g., Taub \& Emurian, 1976), most of the data marshalled in support of the view that human subjects can be trained to control their surface body temperature come from clinical case histories. Although the first reports have been encouraging, controlled experiments must be undertaken to substantiate the early claims and isolate the principles governing the development and loss of such behavior. For this reason, the present study was carried out. The aim was twofold: (1) to see if volunteer college students could be trained to change the surface temperature of their right hand under controlled laboratory conditions and, if so, (2) to determine whether the acquisition of such behavior developed as a specific response or as part of a more comprehensive pattern of surface temperature change.

\section{METHOD}

Eighteen volunteer college students between the ages of 18 and 28 were used as subjects. Females were excluded in order to avoid the confounding influence of estrus on body temperature. All were free of medication.

The apparatus consisted of an E \& M physiograph coupled to two multichannel Yellow Springs telethermometers (Model 41 TD), and a programmed electro-sphygmomanometer. Thermistors were placed on the dorsal surface of each hand, the ventral surface of each ankle, the forehead, under the right arm, and on the ventral surface of the thorax. Blood pressure was recorded from the left arm, and heart rate and respiration were recorded by placing plate electrodes in the fourth intercostal space on each side of the body. Training was carried out over two successive 1-h daily sessions, and feedback consisted of a digital display, the value of which varied inversely with surface temperature of the right hand.

Prior to the onset of the experiment, the subjects were divided randomly into three groups. Those in Group 1 were assigned to the INCREASE condition, those in Group 2 were assigned to the DECREASE condition, and those in Group 3 served as controls. All subjects were instructed to increase and decrease the values on the illuminated digital counter in front of them. In the case of Group 1, an increase in surface tempera-

Please direct reprint requests to S. Thomas Elder, Department of Psychology, University of New Orleans, Lakefront, New Orleans, Louisiana 70122. ture of the right hand was reflected as a decrease in the value of the digital readout; in the case of Group 2, a decrease in hand temperature was accompanied by an increase in the value of the digital display; and in the case of Group 3, surface temperature and digital readout were noncontingent. To control for the possibility that subjects might learn to change the surface temperature of their hand by holding their fingers together to reduce heat loss and spreading them apart to increase heat loss, a small sponge was folded and positioned between adjacent fingers.

\section{RESULTS}

The results (see Figure 1) showed that subjects could learn to raise and lower $[\mathrm{F}(8,60)=20.729, \mathrm{p}<.01]$ the surface temperature of their right hand under conditions of contingent feedback/reinforcement. There was evidence $[F(8,60)=5.518, p<.01]$ of a concomitant change in the surface temperature of the left hand (see Figure 2), but no evidence of systematic temperature changes at other body sites. No changes in heart rate and respiration were observed, but some small uncorrelated changes in blood pressure were found. These data suggest that acquired increases and decreases in surface body temperature are somewhat specific to the site at which there is a response-feedback contingency. The

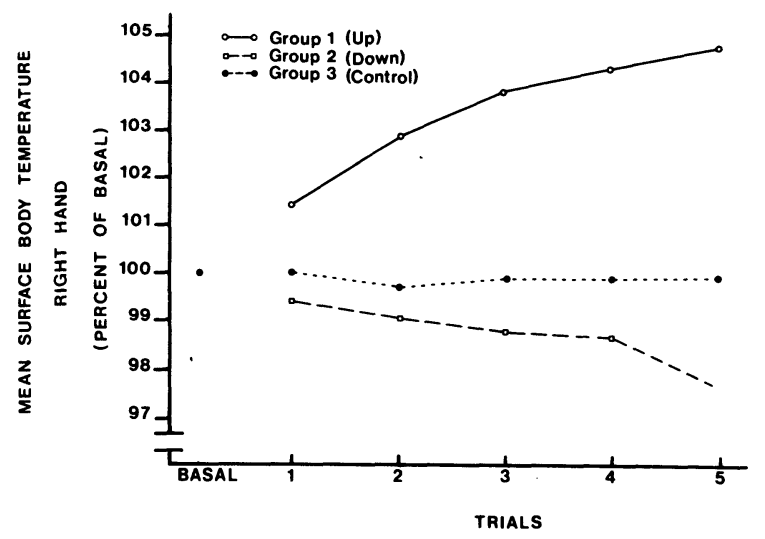

Figure 1. Plot of mean surface body temperature of right hand expressed as percent of basal across groups over trials. 


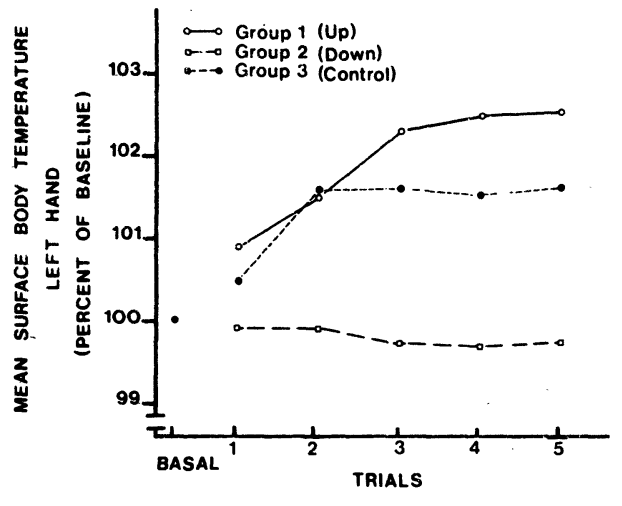

Figure 2. Plot of mean surface body temperature of left hand expressed as percent of basal across groups over trials.

observation that a change in temperature of the right hand was accompanied by a correlated change in surface temperature of the left hand without concomitant changes in forehead, thoracic, or leg temperatures suggests the response was specific along a left-right axis.

\section{DISCUSSION}

The results, though not as dramatic as might be expected from previous reports, showed that subjects could learn to raise and lower the surface temperature of their right hand under conditions of contingent feedback/reinforcement. There was evidence of a concomitant change in the surface temperature of the left hand, thus indicating a patterning effect along a left-right axis. No changes in heart rate and respiration were observed, nor were consistent changes in blood pressure found. Thus, it appears there are a number of characteristics of acquired temperature control which must be taken into account when clinical applications are considered.

\section{REFERENCES}

TAUb, E., \& Emurian, C. S. Feedback-aided self-regulation of skin temperature with a single feedback locus. Biofeedback and Self-Regulation, 1976, 1, 147-168.

(Received for publication March 20, 1978.) 